\title{
New Message Difference for MD4
}

\author{
Yu Sasaki, Lei Wang, Kazuo Ohta, and Noboru Kunihiro \\ The University of Electro-Communications \\ Chofugaoka 1-5-1, Chofu-shi, Tokyo, 182-8585, Japan \\ \{yu339, wanglei, ota, kunihiro\}@ice.uec.ac.jp
}

\begin{abstract}
This paper proposes several approaches to improve the collision attack on MD4 proposed by Wang et al. First, we propose a new local collision that is the best for the MD4 collision attack. Selection of a good message difference is the most important step in achieving effective collision attacks. This is the first paper to introduce an improvement to the message difference approach of Wang et al., where we propose a new local collision. Second, we propose a new algorithm for constructing differential paths. While similar algorithms have been proposed, they do not support the new local collision technique. Finally, we complete a collision attack, and show that the complexity is smaller than the previous best work.
\end{abstract}

Keywords: Hash Function, Collision Attack, MD4, Local Collision, Message Difference, Differential Path.

\section{Introduction}

Hash functions play an important role in modern cryptology. Hash functions must hold the property of collision resistance. This means that it must be computationally hard to find a pair of messages $M$ and $M^{\prime}$ such that $H(M)=H\left(M^{\prime}\right)$ and $M \neq M^{\prime}$.

MD4 is a hash function that was proposed by Rivest in 1990 [5]. MD4 has the Merkle-Damgård structure, and is designed for fast calculation. MD4 has been used to design other hash functions such as MD5 or SHA-1, which are widely used today. Therefore, cryptanalysis on MD4 is important since it affects the cryptanalysis of other hash functions based on MD4.

Several papers have found and reported the weaknesses of MD4. In 1996, the first collision attack was proposed by Dobbertin 1. This attack finds a collision with probability of $2^{-22}$. In 1998, Dobbertin pointed out that the first two rounds of MD4 did not have an property of one way. In 2004, at the CRYPTO rump session, Wang presented that collisions of MD4 could be generated very rapidly [7. In 2005, Wang et al. reported the details of this attack which finds a collision with probability of $2^{-2}$ to $2^{-6}$, and its complexity is less than $2^{8}$ MD4 operations. This attack is highly efficient and many papers have suggested further improvements to this attack. In 2005, Naito et al. presented an improved attack 4. They pointed out the mistakes of the sufficient conditions detailed in [8], and made improvements to the message modification techniques. This

A. Biryukov (Ed.): FSE 2007, LNCS 4593, pp. 329 348 2007.

(C) International Association for Cryptologic Research 2007 
attack finds a collision with complexity less than 3 MD4 operations, and it is the fastest approach up to this time. In 2006, Schläffer and Oswald [6] analyzed how message differences given by 8 , worked, and proposed an automated differential path search algorithm. As a result, they found a differential path that needed fewer sufficient conditions than Wang et al.'s.

An important characteristic of previous attacks is that they all used the same local collision, i.e. the message differences and differential path as proposed by Wang et al. In [8], the authors claim that their message differences, which are derived from their local collision, are optimized for collision attack. However, our research shows that local collision of [8] is not optimized.

For collision attacks, selecting good message differences is very important. Message differences are derived from a local collision, so selecting a good local collision is an important aspect of this work. If better local collisions or message differences are found, attack complexity can be drastically reduced. Moreover, an effective way to find a good local collision or message difference for MD4 may be applicable to other hash functions. This fact motivated our research on MD4.

This paper makes two contributions.

1. We propose a new local collision and new message differences that appear to be the best for collision attack on MD4. We use less than one half of the non-negligible sufficient conditions for new differences needed by Wang et al.'s approach.

2. We show that the differential path construction algorithm proposed by 6] does not work for the new local collision technique. Therefore, we analyze the problems of [6], and develop a new algorithm.

The improved collision attack is realized by combining the above two contributions and the message modification technique described by 34. Since local collision and message differences are improved, we can find a collision with complexity less than 2 MD4 operations, which is the fastest of all existing attacks. We show the new local collision in the right side of Table 1 and Figure 2.2. new message differences and differential path in Table 7 new sufficient conditions in Table 8 and all message modification procedures in Table 9 to Table 20 an example of a collision generated by our attack is shown in Table 4.

The organization of this paper is as follows. In section 2, we explain the specification of MD4 and related works. In section 3, we explain why the local collision of [8] is not best, and propose a new local collision for MD4. In section 4, we explain why the algorithm of [6] does not support the new local collision, and propose a new algorithm that constructs differential paths for the first round of MD4. Section 5 completes the improved attack, and compares its efficiency to previous works. Finally, we conclude this paper in section 6 .

\section{Preliminaries}

\subsection{Specification of MD4 [5]}

MD4 input is an arbitrary length message $M$, and MD4 output is 128-bit data $H(M)$. MD4 has a Merkle-Damgård structure. First, the input message is padded 
to be a multiple of 512 bits. Since padding does not affect collision attack, we omit its explanation. The padded message $M^{*}$ is divided into 512-bit messages $M^{*}=\left(M_{0}, \ldots, M_{n-1}\right)$. The initial value (IV) for the hash value is set to be $H_{0}=(0 \times 67452301$, 0xefcdab89, 0x98badcfe,0x10325476). The output of the compression function $H_{1}$ is calculated using $M_{0}$ and $H_{0}$. In this paper, we call the calculation performed in a single run of the compression function 1 block. Next, $H_{2}$ is calculated using $M_{1}$ and $H_{1}$. Similarly, the compression function is calculated until the last message $M_{n-1}$ is used. Let $H_{n}$ be the hash value of $M$.

\section{Compression Function of MD4}

All calculations in the compression function are 32-bit. We omit the notation of "mod $232 "$. The input to the compression function is a 512-bit message $M_{j}$ and a 128-bit value $H_{j}$. First, $M_{j}$ is divided into $\left(m_{0}, \ldots m_{15}\right)$, where each $m_{i}$ is a 32 bit message. The compression function consists of 48 steps. Steps 1-16 are called the first round (1R). Steps 17-32 and 33-48 are the second and third rounds (2R and $3 \mathrm{R})$, respectively. In step $i$, chaining variables $a_{i}, b_{i}, c_{i}, d_{i}(1 \leq i \leq 48)$ are updated by the following expression $\left(a_{0}, b_{0}, c_{0}, d_{0}\right.$ are the IV).

$a_{i}=d_{i-1}$,

$b_{i}=\left(a_{i-1}+f\left(b_{i-1}, c_{i-1}, d_{i-1}\right)+m_{k}+t\right) \lll s_{i}$,

$c_{i}=b_{i-1}$,

$d_{i}=c_{i-1}$,

where $f$ is a Boolean function defined in each round, $m_{k}$ is one of $\left(m_{0}, \ldots m_{15}\right)$, and index $k$ is defined in each step. If $m_{0}, \ldots m_{15}$ are fixed, all other $m_{i}(16 \leq$ $i \leq 47)$ are also fixed. In this paper we call this "Message Expansion". $t$ is a constant number defined in each round, $\lll s_{i}$ denotes left rotation by $s_{i}$ bits, and $s_{i}$ is defined in each step. Details of $f$ and $t$ are as follows.

$$
\begin{aligned}
& 1 R: t=0 x 00000000, f(X, Y, Z)=(X \wedge Y) \vee(\neg X \wedge Z), \\
& 2 R: t=0 x 5 a 827999, f(X, Y, Z)=(X \wedge Y) \vee(Y \wedge Z) \vee(X \wedge Z), \\
& 3 R: t=0 x 6 e d 9 e b a 1, f(X, Y, Z)=X \oplus Y \oplus Z
\end{aligned}
$$

After 48 steps are calculated, $H_{j+1}$ is calculated as follows.

$$
\begin{aligned}
& a a_{0} \leftarrow a_{48}+a_{0}, \quad b b_{0} \leftarrow b_{48}+b_{0}, \\
& c c_{0} \leftarrow c_{48}+c_{0}, \quad d d_{0} \leftarrow d_{48}+d_{0}, \\
& H_{j+1} \leftarrow\left(a a_{0}\left|b b_{0}\right| c c_{0} \mid d d_{0}\right) .
\end{aligned}
$$

\subsection{Related Work 1: Collision Attack by Wang et al. and Its Improvement by Previous Works}

The first collision attack on MD4 was proposed by Wang et al. in 2005. Many researchers have improved this attack since its publication. Our paper also improves this attack. In this section, we explain the attack procedure and which part of the attack has been improved so far.

This attack is a differential attack, and generates a collision with complexity less than $2^{8}$ MD4 operations. Let $m$ and $m^{\prime}$ be a pair of messages that yield a collision. Difference $\Delta$ is defined to be the value yielded by subtracting value for 
$m$ from value for $m^{\prime}$. For example, $\Delta m=m^{\prime}-m$. The attack procedure is as follows.

1. Find the "Message Difference $(\Delta M)$ " that yields a collision with high probability.

2. Determine how the impact of $\Delta M$ propagates. The propagation of this difference is called the "Differential Path (DP)."

3. Generate "Sufficient Conditions (SC)" for realizing the differential path on the value of chaining variables for calculating the hash value of $m$.

4. Determine the procedures of "Message Modification (MM)" that satisfy sufficient conditions.

5. Locate a message that satisfies all sufficient conditions by randomly generating messages and applying Message Modification. Let such a message be $M_{*}$.

6. Calculate $M_{*}^{\prime}=M_{*}+\Delta M$. Finally, $M_{*}$ and $M_{*}^{\prime}$ become a collision pair.

$\Delta M$ of Wang et al.'s attack is as follows.

$$
\begin{gathered}
\Delta M=M^{\prime}-M=\left(\Delta m_{0}, \Delta m_{1}, \ldots, \Delta m_{15}\right) \\
\Delta m_{1}=2^{31}, \Delta m_{2}=2^{31}-2^{28}, \Delta m_{12}=-2^{16}, \Delta m_{i}=0,0 \leq i \leq 15, i \neq 1,2,12 .
\end{gathered}
$$

[8] introduces two kinds of message modification: Single-Step Modification and Multi-Step Modification. Later, these names were changed to Basic Message Modification (BMM) and Advanced Message Modification (AMM). They are related as follows.

- Basic message modification is the technique that can satisfy all sufficient conditions in the first round with probability of 1.

- Advanced message modification can satisfy several sufficient conditions in the second round. (If the sufficient conditions exist in an early step of the second round, they may be satisfied by advanced message modification. On the other hand, if they exist in a later step of the second round, it's almost impossible to satisfy them.)

- No technique is known to satisfy any sufficient conditions in the third round.

For details about the differential path, sufficient condition and message modification, please refer to 8 .

\section{Strategy of Selecting $\Delta M$}

The strategy of selecting $\Delta M$ is explained by Schläffer and Oswald [6]. They showed that $\Delta M$ was determined by the Local Collision (LC) in the third round, although the reason was not explained. They then showed how Wang et al.'s local collision worked. We show the local collision of Wang et al. in the left side of Table 1, and its diagram in Figure 2.2.

\section{Improvement of Collision Attack}

So far, several papers 4,6] have improved the collision attack proposed by Wang et al. Naito et al. 4] pointed out the sufficient condition mistakes in [8], and made improvements to message modification. They used the same $\Delta M$, differential 
Table 1. Comparison of Wang et al.'s Local Collision and Ours

\begin{tabular}{|c|cccc|c|}
\hline \multicolumn{5}{|c|}{ Wang et al.'s Local Collision } \\
\hline Step & $\Delta a_{k}$ & $\Delta b_{k}$ & $\Delta c_{k}$ & $\Delta d_{k}$ & $\Delta m_{k-1}$ \\
\hline$i$ & & $2^{31}$ & & & $2^{31-s_{i}}$ \\
$i+1$ & & $2^{31}$ & $2^{31}$ & & $2^{31-s_{i+1}}, 2^{31}$ \\
$i+2$ & & & $2^{31}$ & $2^{31}$ & \\
$i+3$ & $2^{31}$ & & & $2^{31}$ & \\
$i+4$ & $2^{31}$ & & & & \\
$i+5$ & & & & $2^{31}$ \\
\hline
\end{tabular}

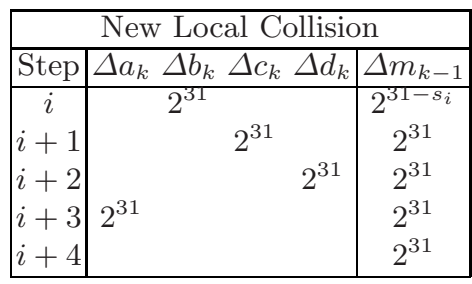
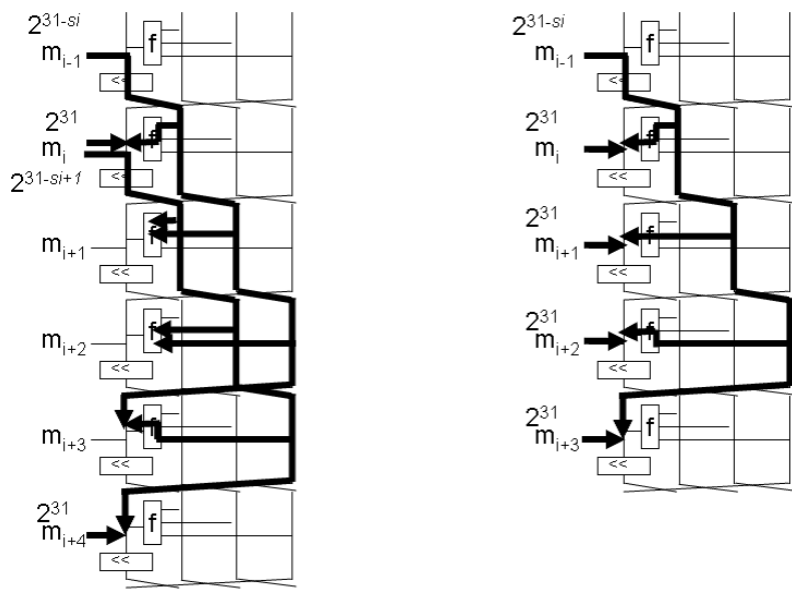

Fig. 1. Diagram of Wang et al.'s Local Collision and Ours

path and sufficient condition as given by [8], but proposed advanced message modification that could satisfy all sufficient conditions in the second round and quickly satisfy sufficient conditions in the third round 1 . Their attack finds a collision of MD4 with complexity less than 3 MD4 operations. Schläffer and Oswald 6] proposed an algorithm that could construct differential paths when $\Delta M$ was given. They used the same $\Delta M$ as given by [8], and showed a differential path that needed fewer sufficient conditions than Wang et al.'s.

For Wang et al.'s attack, selection of $\Delta M$ gives the most significant impact to the attack complexity. However, no paper has attempted to improve the $\Delta M$ of Wang et al. In this paper, we propose new $\Delta M$ in section 3.

\subsection{Related Work 2: Differential Path Search by Schläffer and Oswald 6.}

At FSE06, Schläffer and Oswald [6] proposed an algorithm on how to construct differential paths for MD4 collision attack, when message differences are given.

${ }^{1}$ This technique is restarting collision search from an intermediate step, which is different from message modification for the third round. 
They used Wang et al.'s message differences. We show this algorithm can not be applied to the new $\Delta M$ (see section 4.2 and 4.3 ). In this section, we explain their algorithm as follows.

1. Calculate "Target Differences." In step $i$, target differences mean differences that are used to cancel $\Delta m$ in steps $i+4 k(k=0, \pm 1, \pm 2 \cdots)$.

2. Determine the actual output differences of $f$ function. $f$ function can not produce differences that are not elements of target differences. Resulted differential path usually has some contradictions in sufficient conditions.

3. Resolve contradictions in the sufficient conditions to construct a differential path.

For more details, please refer to [6].

\section{New Local Collision}

As mentioned in section 2.2, no paper has proposed a better $\Delta M$ than that of Wang et al. In this section, we propose a superior local collision and $\Delta M$ that are the best for collision attack on MD4.

\subsection{Importance of Selecting $\Delta M$}

6] determined $\Delta M$ was set by local collision in the third round, however, they didn't mention a reason for this. This motivated us to start with an explanation.

Thanks to the various message modification techniques which are available to us, following the differential path during the first two rounds (32 steps) of MD4 has very low cost. The computational cost of the attack comes from the probability of following the path during the third round. As a consequence, in order to get a more efficient collision attack on MD4, minimizing the number of sufficient conditions in the third round is the most important. Therefore, we start by looking for a very efficient local collision in the third round.

\subsection{Problem of Wang et al.'s Local Collision and Constructing New Local Collision}

In order to minimize the number of sufficient conditions in the third round, the method of Wang et al. applies local collision there. Their local collision is shown in left side of Table 1. They insert single message difference to step $i$ in the third round, and insert other message differences to minimize the impact of the propagation of the inserted difference. As a result, the inserted difference is cancelled in following 6 steps. In Wang et al.'s local collision, two differences are made in the MSB of $b_{i}$ and $b_{i+1}$ for ensuring that differences don't propagate in step $i+2, i+3$ and $i+4$ (obviates the need to insert other message differences). Here, due to the addition and left rotation, the following fact is achieved. (We show the proof of this in Appendix A.) 
Fact: To make $\Delta b_{i}= \pm 2^{31}$ from $\Delta m_{k}= \pm 2^{31-s_{i}}$, we need the following sufficient condition:

$$
\begin{cases}b_{i, 31}=0 & \left(\text { if } \Delta m_{k}=+2^{31-s_{i}}\right) \\ b_{i, 31}=1 & \left(\text { if } \Delta m_{k}=-2^{31-s_{i}}\right)\end{cases}
$$

Wang et al.'s local collision makes two differences. Therefore, it needs two sufficient conditions to realize the local collision.

In a collision attack on MD4, at least one message difference in the third round is necessary since collision messages must be different. Considering the Fact, it is obvious that if a local collision yielding one difference in the MSB of $b_{i}$ can be constructed, that local collision is the best.

\subsection{Construction of New Local Collision}

We construct a new local collision by making only one difference in the MSB of $b_{i}$. The new local collision is summarized in right side of Table 1 and Figure 2.2 In step $i$, we make $\Delta b_{i}= \pm 2^{31}$ by inserting $\pm 2^{31-s_{i}}$ on $m_{i-1}$. From the Fact, we need one sufficient condition to make $\Delta b_{i}= \pm 2^{31}$. In step $i+1, \pm 2^{31}$ of $b_{i}$ propagates through function $f=\left(b_{i} \oplus c_{i} \oplus d_{i}\right)$. Therefore, we insert $\pm 2^{31}$ on $m_{i}$ in order to cancel $\pm 2^{31}$ from $f$. A similar situation occurs in step $i+2$ and $i+3$, so we insert $\pm 2^{31}$ on $m$, to cancel the difference that propagates from $f$. In step 4 , we cancel $\Delta a_{i+3}= \pm 2^{31}$ by $\Delta m_{i+3}= \pm 2^{31}$, and finally all differences are cancelled. (Since all $\Delta m$ are in MSB, any sign for $\Delta m$ is acceptable.)

\subsection{New Message Difference}

As described in section 3.3, the new local collision makes only one difference, therefore, the new local collision needs only one sufficient condition in the third round, and this is the best local collision for MD4. In order to complete the entire collision attack, the impact of the message expansion on the previous rounds needs to be considered. The local collision constructed in section 3.3 does not fix step $i$ that is the initial step of the local collision. Therefore, we analyze the impact of message expansion, and select the best $i$ that minimizes the impact on the previous rounds.

We show details of this analysis in Appendix B. As a result, we found that $i=33$ is the best, and obtained following message differences.

$$
\begin{aligned}
& \Delta m_{0}=2^{28}, \Delta m_{2}=2^{31}, \Delta m_{4}=2^{31}, \Delta m_{8}=2^{31}, \Delta m_{12}=2^{31}, \\
& \Delta m_{i}=0 \text { for } i=1,3,5,6,7,9,10,11,13,14,15 .
\end{aligned}
$$

The differential path for the third round is also determined by local collision. It is shown in Table 7 in Appendix C.

Remaining work is to construct a differential path for the first and second round. In the first round, all sufficient conditions are satisfied by basic message modification, whereas, some of sufficient conditions in the second round may not 
be satisfied by advanced message modification. Therefore, we should minimize the number of sufficient conditions in the second round even if the differential path for the first round becomes more complicated. Since constructing the differential path for the second round is simple, we show only the result in Table 7 in Appendix C.

\section{Differential Path Search Algorithm}

In this section, we describe the DP construction algorithm, denoted as DPC.

\subsection{Definition of Good $D P C$}

Input of $D P C$ is $\triangle M$. A good $D P C$ should not just work for one specified $\triangle M$. It should be able to work for all $\Delta M$. Moreover, it should be finished in reasonable time. If a $D P C$ is able to work for all $\Delta M$ in reasonable time, we will call it "Good $D P C . "$

\section{Crucial technique to Realize Good $D P C$}

For DPC, how to control difference propagation through $f$ function is important. Therefore, in design of good $D P C$, it is crucial to control difference propagation through $f$ function to search DP in a large space efficiently.

\subsection{Overview of Problems of Previous Work [6] and Our Improvement}

Schläffer and Oswald proposed an algorithm to construct differential paths, based on Wang et al.'s message differences. Unfortunately the algorithm can not work for our new message differences. Therefore, we will propose a new $D P C$, which is able to work for our new $\Delta M$. We will give an overview of comparison of these two algorithms.

\section{Problem of Schläffer and Oswald's Algorithm}

By Schläffer and Oswald's algorithm, the differences produced by $f$ function in step $i$ can only be used in step $i+4 k$, and no others. Although their rule reduced the search space considerably, they found, fortunately, a better differential path than Wang et al's algorithm. However, reduced search space is too small, and consequently, their algorithm can not work for our new message differences.

\section{Advantage of Our Algorithm}

In our algorithm, we found a way to control difference propagation through $f$ function efficiently in larger search space.

Important improvement: for step $i$, Schläffer and Oswald's algorithm only produces difference of $f$ to cancel $\Delta M$ in step $i+4 k$. By our algorithm, besides this kind of difference, $f$ can also produce difference to guarantee that $\Delta M$ in other steps can be cancelled.

The search space of our algorithm is larger, which makes our algorithm work for the new $\Delta M$ proposed in section 3 . 


\subsection{Details of Our New Proposed Algorithm for the First Round}

The goal of our algorithm is to construct a differential path for the first round with given chaining variable differences after step 16 and given message differences. Our algorithm consists of three major parts: Forward search, Backward search and Joint algorithm.

\section{i. Forward search}

Forward search is done from step 1 to step 4. As mentioned in section 4.1, the crucial technique for making good $D P C$ is controlling difference propagation through $f$ function in a large search space. In forward search, we exhaustively search the possibility of difference through $f$. After forward search is finished, there are possible differences for chaining variables after step 4. Possible differences for $a_{4}, b_{4}, c_{4}, d_{4}$ are called "Potential Differences."

\section{ii. Backward search}

First of all, we will redefine the concept "Target Difference" for backward search. Backward Search is done from step 16 to step 8.

Target Differences: for step $i$, target differences $\Delta t_{i}$ are calculated by differences of chaining variable $b_{i}$ and message differences $\Delta m_{i-1}$ as follows:

$$
\Delta t_{i}=-\Delta m_{i-1}+\left(\Delta b_{i} \ggg s_{i}\right)(8 \leq i \leq 16)
$$

Chaining variable differences after step 16 are already fixed, in other words, differences of $b_{i}(12 \leq i \leq 16)$ are fixed, it is easy to calculate target differences from step 16 to step 12 . In step $i$, a target difference can be achieved by differences of $a_{i-1}$ or $f$ function. We need to determine each target difference which should be produced by $a_{i-1}$ or $f$ function. During backward search, we assume that differences of $a_{i-1}$ and input differences of $f$ function can always be produced in previous steps, so any target difference can be produced by $a_{i-1}$. This is main idea of Schläffer and Oswald's algorithm. We enlarge the search space by considering how to guarantee target differences produced by $f$ function.

If we determine that a target difference will be achieved by $f$, input chaining variables $b_{i-1}, c_{i-1}$, or $d_{i-1}$ should have a difference at the same position. If $b_{i-1}, c_{i-1}$, or $d_{i-1}$ has a difference, it should be cancelled in the following step. If it can not be cancelled, it can not be used.

Considering that the chaining variable differences after step 16 are already fixed, from step 16 to step 12, it is very easy to check whether a difference of $b_{i-1}, c_{i-1}$ or $d_{i-1}$ can be cancelled in the following steps.

After backward search from step 16 to step 12 is done, there are some candidates for chaining variable differences in step 12 . For every candidate, backward search from step 12 to step 8 is done by applying the same method.

After backward search from step 16 to 8 is done, there are some candidates for chaining variable differences in step 8, which are called "Aimed Differences."

\section{iii. Joint algorithm}

From step 4 to step 8, aimed differences will select potential differences. In the joint algorithm, only potential differences that match aimed differences can be 
produced by $f$ function. Selected potential differences need to be cancelled in the following steps, To cancel these differences, carry can be used to expand input differences of $f$ function. More differences can also be introduced.

After all the selected differences are canceled, that is, after the joint algorithm is finished, a differential path is constructed.

\section{Attack Implementation}

\subsection{Message Modification}

The results of Section 3 and 4 yield the new $\Delta M$, differential path and sufficient conditions. To complete the collision attack, what remains is to propose advanced message modification procedures for satisfying sufficient conditions in the second and third round. Therefore, we propose advanced message modification, and evaluate the total attack complexity of proposed attack. Papers [34] give some good ideas of advanced message modification. Since proposing advanced message modification is outside the scope of this paper, we show only the procedures of advanced message modification in Table 9 to Table 20 in Appendix D. As a result of applying advanced message modification, all sufficient conditions in the second round are satisfied with very high probability. Furthermore, by applying the technique called "Shortcut Modification" by [4] or "Tunnel" by [3], the sufficient condition in the third round is also quickly satisfied.

\subsection{Attack Procedure and Complexity Estimation}

The attack procedure is shown in Table 2. (Note extra conditions are set for advanced message modification. These are introduced in Appendix D.)

For line 1 to 5: Computation for $m_{i}$ needs almost 1 MD4 step. Therefore, for line 4 , we need $16 \mathrm{MD} 4$ steps. Line 2 and 3 can be done very quickly compared to line 4 .

For line 6 to 13: For line 7, computation for $b_{i}$ is 1 MD4 step. Therefore, for line 7 , we need 13 MD4 steps. For line 10, there are 11 message modification procedures. According to our evaluation, each procedure requires less than 3 MD4 steps (See Table 9 to 19 for details). Since each condition is satisfied with probability $1 / 2$, we expect half of the message modification procedures are executed. Therefore, we need $3 \times 11 \div 2=16.5$ MD4 steps.

For line 14 to 18: Line 14 needs 4 steps of MD4 operations. Then, if " $b_{33,31}=0$ " is not satisfied, we apply message modification. According to our evaluation, modifying message for " $b_{33,31}=0$ " takes less than 1 MD4 step, and the probability that this condition is satisfied after repeating line 14 to 16 is $1 / 2$. Therefore, this condition is expected to be satisfied at most 2 trial. Complexity of satisfying in the first trial is $4 \mathrm{MD} 4$ steps and by the second trial is $4+4+1=9 \mathrm{MD} 4$ steps. Therefore, average complexity is $(4+9) \div 2=6.5$ MD4 steps. Complexity for line 18 is negligible. 
Table 2. Attack Procedure

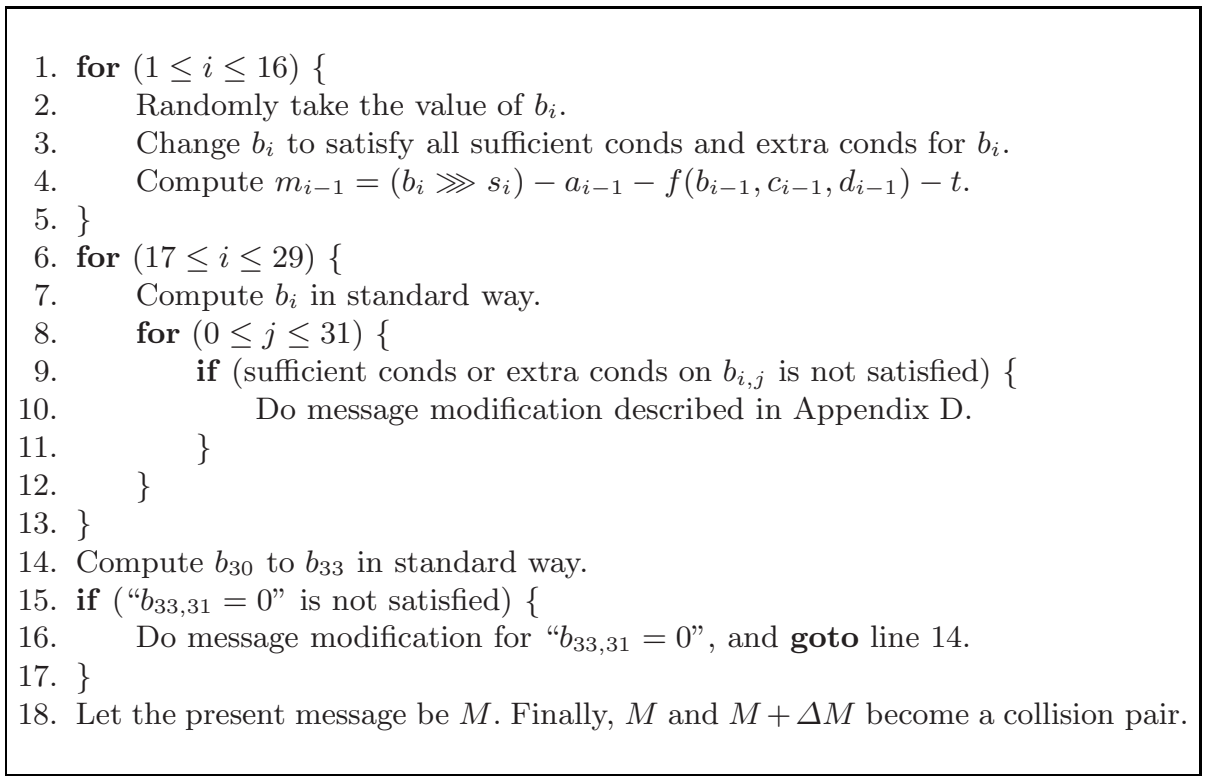

Total complexity: Total complexity is addition of above three parts, that is, $16+13+16.5+7.5=53 \mathrm{MD} 4$ steps. Considering other trivial complexity, we evaluate that the complexity of our attack is less than 2 MD4 operations(=96 steps).

\subsection{Comparison with Previous Attacks}

The efficiencies of the proposed attack and previous attacks are compared in Table 3 .

Table 3. Comparison of Previous Attacks and Our Result

\begin{tabular}{|c|c|c|c|c|}
\hline Method & $\Delta M$ & $\begin{array}{c}\text { \#SCs } \\
\text { in 2R }\end{array}$ & $\begin{array}{c}\text { \#SCs } \\
\text { in 3R }\end{array}$ & $\begin{array}{c}\text { Total complexity } \\
\text { (Unit MD4 operations) }\end{array}$ \\
\hline Wang et al. & Wang's $\Delta M$ & 25 & 2 & Less than $2^{8}$ \\
\hline Naito et al. & Wang's $\Delta M$ & 25 & 2 & Less than 3 \\
\hline Schläffer and Oswald & Wang's $\Delta M$ & 22 & 2 & - \\
\hline Proposed attack & $\Delta M$ for new LC & 9 & 1 & Less than 2 \\
\hline
\end{tabular}

From Table 3, it can be said that improvement of local collision is very important. With the new local collision, number of sufficient conditions in the second and third round becomes less than half those of previous attacks, and this enables us to further reduce the complexity of generating a collision. 


\section{Conclusion}

This paper proposed a new local collision that is the best for collision attack on MD4. This was achieved by the first improvement to the message difference approach of Wang et al.

For successful collision attacks, $\Delta M$ must be carefully selected to reduce the number of sufficient conditions that cannot be satisfied by message modification. In this paper, we showed how efficient the proposed improvement to $\Delta M$ is. With the new $\Delta M$, number of sufficient conditions in the second and third round is less than half those of previous studies; attack complexity is also improved.

Improving the attack on MD4 is not the final goal. As future works, we will apply our analysis to other hash functions, and aim to find more efficient $\Delta M$.

We realized that differential path construction algorithm proposed by [6] did not work with the new $\Delta M$. Therefore, we analyzed the problems of [6], and proposed a new algorithm for constructing differential path for the first round.

Finally, we show a collision generated by using the new local collision in Table 4. We note that $\Delta M$ of this example is different from all previous works.

Table 4. An Example of MD4 Collision Generated with New Local Collision

\begin{tabular}{|c|c|}
\hline $\bar{M}$ & 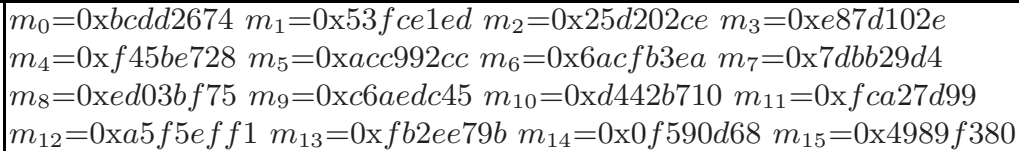 \\
\hline$M^{\prime}$ & $\begin{array}{l}m_{0}^{\prime}=0 \mathrm{x} c c d d 2674 m_{1}^{\prime}=0 \times 53 f c e 1 e d m_{2}^{\prime}=0 x a 5 d 202 c e m_{3}^{\prime}=0 x e 87 d 102 e \\
m_{4}^{\prime}=0 x 745 b e 728 m_{5}^{\prime}=0 x a c c 992 c c m_{6}^{\prime}=0 x 6 a c f b 3 e a m_{7}^{\prime}=0 x 7 d b b 29 d 4 \\
m_{8}^{\prime}=0 x 6 d 03 b f 75 m_{9}^{\prime}=0 x c 6 a e d c 45 m_{10}^{\prime}=0 x d 442 b 710 m_{11}^{\prime}=0 x f c a 27 d 99 \\
m_{12}^{\prime}=0 x 25 f 5 \text { eff } 1 m_{13}^{\prime}=0 x f b 2 e e 79 b m_{14}^{\prime}=0 x 0 f 590 d 68 m_{15}^{\prime}=0 \times 4989 f 380\end{array}$ \\
\hline & 0x c257b7be 324f26ef $69 d 3 d 290$ b01be001 \\
\hline
\end{tabular}

\section{Acknowledgements}

We would like to thank Antoine Joux for improving our paper and anonymous reviewers for helpful comments.

\section{References}

1. Dobbertin, H.: Cryptanalysis of MD4. In: Gollmann, D. (ed.) Fast Software Encryption. LNCS, vol. 1039, pp. 53-69. Springer, Heidelberg (1996)

2. Dobbertin, H.: The First Two Rounds of MD4 are Not One-Way. In: Vaudenay, S. (ed.) FSE 1998. LNCS, vol. 1372, pp. 284-292. Springer, Heidelberg (1998)

3. Klima, V.: Tunnels in Hash Functions: MD5 Collisions Within a Minute. Cryptology ePrint Archive, Report (2006)/105

4. Naito, Y., Sasaki, Y., Kunihiro, N., Ohta, K.: Improved Collision Attack on MD4 with Probability Almost 1. In: Won, D.H., Kim, S. (eds.) ICISC 2005. LNCS, vol. 3935, pp. 129-145. Springer, Heidelberg (2006) 
5. Rivest, R.: The MD4 Message Digest Algorithm. In: Menezes, A.J., Vanstone, S.A. (eds.) CRYPTO 1990. LNCS, vol. 537, pp. 303-311. Springer, Heidelberg (1991), http://www.ietf.org/rfc/rfc1320.txt

6. Schläffer, M., Oswald, E.: Searching for Differential Paths in MD4. In: Robshaw, M. (ed.) FSE 2006. LNCS, vol. 4047, pp. 242-261. Springer, Heidelberg (2006)

7. Wang, X., Feng, D., Chen, H., Lai, X., Yu, X.: Collision for Hash Functions MD4, MD5, HAVAL-128 and RIPEMD. In: Franklin, M. (ed.) CRYPTO 2004. LNCS, vol. 3152, Springer, Heidelberg (2004)

8. Wang, X., Lai, X., Feng, D., Chen, H., Yu, X.: Cryptanalysis of the Hash Functions MD4 and RIPEMD. In: Cramer, R.J.F. (ed.) EUROCRYPT 2005. LNCS, vol. 3494, pp. 1-18. Springer, Heidelberg (2005)

\section{A Proof of the Fact in Section 3.2}

Proof. Remember that the calculation of $b_{i}$ in step $i$ is as follows.

$$
b_{i}=\left(a_{i-1}+f\left(b_{i-1}, c_{i-1}, d_{i-1}\right)+m_{k}+t\right) \lll s_{i}
$$

Here, we make $\Delta b_{i}=2^{31}$ or $-2^{31}$ by adding the difference $\Delta m_{k}=+2^{31-s_{i}}$ or $-2^{31-s_{i}}$. Let the value of $a_{i-1}+f\left(b_{i-1}, c_{i-1}, d_{i-1}\right)+m_{k}+t$ be $\Sigma$. We focus on the value of $\Sigma$ in bit position $31-s_{i}$ (In short, $\Sigma_{31-s_{i}}$ ). $\Sigma_{31-s_{i}}$ is 0 or 1 . First, we consider the case that $\Sigma_{31-s_{i}}$ is 0 . In this case, if $\Delta m_{k}$ is positive, $\Sigma_{31-s_{i}}$ changes from 0 to 1 , and other bits are kept unchanged. Therefore, it becomes $\Delta b_{i}=+2^{31}$ after the rotation. However, if $\Delta m_{k}$ is negative, $\Sigma_{31-s_{i}}$ changes from 0 to 1 , and bit position $31-s_{i}+1$ also changes because of carry. Here, assume that carry is stopped in bit position $31-s_{i}+1$, therefore, the value in bit position $31-s_{i}+1$ changes from 1 to 0 , and other bits are unchanged. In this case, after the rotation, it becomes $\Delta b_{i}=+2^{31}-2^{0}$, and thus, we cannot obtain the desired difference. The same analysis is applied in the case of $\Sigma_{31-s_{i}}$ is 1 . The result of the analysis is summarized below.

Table 5. Summary of Proof

\begin{tabular}{|c|c|c|}
\hline & $\Delta m_{k}=+2^{31-s_{i}}$ & $\Delta m_{k}=-2^{31-s_{i}}$ \\
\hline$\Sigma_{31-s_{i}}=0$ & Success & Failure \\
\hline$\Sigma_{31-s_{i}}=1$ & Failure & Success \\
\hline
\end{tabular}

Consider the fact that $\Sigma_{31-s_{i}}=b_{i, 31}$, we get following conclusion; To make success, $b_{i, 31}=0$ if $\Delta m_{k}=+2^{31-s_{i}}$, or $b_{i, 31}=1$ if $\Delta m_{k}=-2^{31-s_{i}}$. (Q.E.D)

\section{B Selecting $\Delta M$ by Considering Message Expansion}

As described in section 3.3, the new local collision makes one difference in the third round. This does not fix step $i$ that is the initial step of the local collision. 
Therefore, we analyze the impact of message expansion, and select the best $i$ that minimizes the impact on the previous rounds.

However, thanks to the basic message modification, even if the differential path becomes complicated and many sufficient conditions are required, all sufficient conditions in the first round can be satisfied with probability 1 . Therefore, we only have to care about the impact on the second round.

The third round consists of step 33-48. Since the new local collision takes 5 steps to realize cancellation, we have 12 choices for the initial step of local collision $($ IniLC $),(33 \leq$ IniLC $\leq 44)$. To determine a good IniLC, we consider following characteristics of the second round.

- We need to cancel all differences by the final step of the second round since local collision in the third round must start from no-difference state.

- Let the last step with some message difference in the second round be $c p$, which stands for Cancellation Point. If all differences are cancelled at step $c p$, remaining steps of the second round are guaranteed to be no-difference without sufficient conditions.

Therefore, in order to reduce number of sufficient conditions in the second round, $c p$ should be as early a step as possible. Finally, we select IniLC to minimize $c p$. We show the analysis of message expansion in Table 6. In Table 6 gray highlight shows an example of the analysis for $\operatorname{Ini} L C=33$. Messages that have differences on the second round and third round are colored. From the table, we see that $c p$ for $\operatorname{IniLC}=33$ is 25 . The same analysis is applied for all IniLC. $c p$ for all IniLC are listed in the bottom of Table 6.

Table 6. Analysis of Impact of Message Expansion

Message index for each step in $2 \mathrm{R}$ and $3 \mathrm{R}$

\begin{tabular}{|c|c|c|c|c|c|c|c|c|c|c|c|c|c|c|c|}
\hline \multirow[t]{2}{*}{$2 \mathrm{R}$} & step number & 17 & 18 & 19 & 20 & 21 & 22 & 23 & 24 & 25 & 26 & \begin{tabular}{l|l|}
27 & 28 \\
\end{tabular} & 29 & 30 & \begin{tabular}{l|l|}
31 & 32 \\
\end{tabular} \\
\hline & message in & 0 & 4 & 8 & 12 & 1 & 5 & 9 & 13 & 2 & 6 & \begin{tabular}{|l|l|}
10 & 14 \\
\end{tabular} & 3 & 7 & \begin{tabular}{l|l}
11 & 15 \\
\end{tabular} \\
\hline \multirow[t]{2}{*}{$3 \mathrm{R}$} & & 33 & 34 & 35 & 36 & 37 & 38 & 39 & 40 & 41 & \begin{tabular}{|l|}
42 \\
\end{tabular} & \begin{tabular}{|l|l|}
43 & 44 \\
\end{tabular} & 45 & \begin{tabular}{|l|}
46 \\
\end{tabular} & \\
\hline & essa & 0 & 8 & 4 & 12 & 2 & 10 & 6 & 14 & 1 & 9 & \begin{tabular}{l|l|l}
5 & 13
\end{tabular} & 3 & 11 & \begin{tabular}{l|l}
7 & 15
\end{tabular} \\
\hline
\end{tabular}

The value of $c p$ for all IniLC

\begin{tabular}{|r||l|l|l|l|l|l|l|l|l|l|l|l|}
\hline IniLC & 33 & 34 & 35 & 36 & 37 & 38 & 39 & 40 & 41 & 42 & 43 & 44 \\
\hline$c p$ & 25 & 27 & 27 & 28 & 28 & 28 & 28 & 28 & 29 & 31 & 31 & 32 \\
\hline
\end{tabular}

From Table 6. finally, we find that $\operatorname{IniLC}=33$ is the best since its $c p$ is the smallest. The obtained $\Delta M$ and differential path for the third round is shown in Table 7 in Appendix $\mathrm{d}^{2}$.

\footnotetext{
${ }^{2}$ We also calculate the good $\Delta M$ for Wang et al.'s local collision by using this analysis. Then, we found that the resulting $\Delta M$ is the same as proposed by Wang et al.'s.
} 


\section{Differential Path and Sufficient Conditions}

Table 7. Differential Path of MD4 for New Local Collision

\begin{tabular}{|c|c|c|c|c|}
\hline \multicolumn{2}{|c|}{\begin{tabular}{|l|l|} 
Step & Shift \\
\end{tabular}} & \multirow[b]{2}{*}{$\Delta m_{i-1}$} & \multicolumn{2}{|c|}{$\Delta b_{i}$} \\
\hline$i$ & $s_{i}$ & & Numerical difference & Difference in each bit \\
\hline \multirow[t]{2}{*}{1} & \multirow[t]{2}{*}{3} & \multirow[t]{2}{*}{$2^{28}$} & $-2^{31}$ & $\Delta[-31]$ \\
\hline & & & $-2^{0}$ & $\Delta[0,-1]$ \\
\hline \multirow[t]{2}{*}{2} & \multirow[t]{2}{*}{7} & & $-2^{7}$ & $\Delta[7]$ \\
\hline & & & $-2^{8}$ & $\Delta[-9]$ \\
\hline 3 & 11 & $2^{31}$ & $-2^{11}$ & $\Delta[-11]$ \\
\hline 4 & 19 & & $-2^{19}$ & $\Delta[19,20,-21]$ \\
\hline \multirow[t]{2}{*}{5} & \multirow[t]{2}{*}{3} & \multirow[t]{2}{*}{$2^{31}$} & $2^{3}$ & $\Delta[3]$ \\
\hline & & & $2^{22}$ & $\Delta[-22,-23,24]$ \\
\hline \multirow[t]{2}{*}{6} & \multirow[t]{2}{*}{7} & & $-2^{18}$ & $\Delta[18,19,-20]$ \\
\hline & & & $2^{31}$ & $\Delta[31]$ \\
\hline \multirow[t]{3}{*}{7} & \multirow[t]{3}{*}{11} & & $2^{1}$ & $\Delta[-1, \cdots, 9]$ \\
\hline & & & $-2^{22}$ & $\Delta[22,23,-24]$ \\
\hline & & & $2^{31}$ & $\Delta[31]$ \\
\hline 8 & 19 & & & \\
\hline \multirow[t]{2}{*}{9} & \multirow[t]{2}{*}{3} & \multirow[t]{2}{*}{$2^{31}$} & $2^{22}$ & $\Delta[-22,23]$ \\
\hline & & & $2^{25}$ & $\Delta[25]$ \\
\hline \multirow[t]{3}{*}{10} & \multirow[t]{3}{*}{7} & & $-2^{12}$ & $\Delta[-12]$ \\
\hline & & & $-2^{25}$ & $\Delta[25,26,-27]$ \\
\hline & & & $2^{29}$ & $\Delta[-29,-30,31]$ \\
\hline 11 & 11 & & $2^{12}$ & $\Delta[12]$ \\
\hline 12 & 19 & & $-2^{12}$ & $\Delta[-12]$ \\
\hline \multirow[t]{2}{*}{13} & \multirow[t]{2}{*}{3} & \multirow[t]{2}{*}{$2^{31}$} & $2^{25}$ & $\Delta[25]$ \\
\hline & & & $-2^{28}$ & $\Delta[28,-29]$ \\
\hline 14 & 7 & & & \\
\hline 15 & 11 & & & \\
\hline 16 & 19 & & $-2^{31}$ & $\Delta[-31]$ \\
\hline 17 & 3 & $2^{28}$ & $2^{28}$ & $\Delta[28]$ \\
\hline 18 & 5 & $2^{31}$ & & \\
\hline 19 & 9 & $2^{31}$ & & \\
\hline 20 & 13 & $2^{31}$ & & \\
\hline 21 & 3 & & $2^{31}$ & $\Delta[31]$ \\
\hline 22 & 5 & & & \\
\hline 23 & 9 & & & \\
\hline 24 & 13 & & & \\
\hline 25 & 3 & $2^{31}$ & & \\
\hline$\cdots$ & & & & \\
\hline 33 & 3 & $2^{28}$ & $2^{31}$ & $\Delta[31]$ \\
\hline 34 & 9 & $2^{31}$ & & \\
\hline 35 & 11 & $2^{31}$ & & \\
\hline 36 & 15 & $2^{31}$ & & \\
\hline 37 & 3 & $2^{31}$ & & \\
\hline
\end{tabular}

The symbol ' $\Delta[i]$ ' means the value of chaining variable in bit position $i$ changes from 0 to 1 by difference. The symbol ' $\Delta[-i]$ ' means it changes from 1 to 0 instead. 
Table 8. Sufficient Conditions and Extra Conditions for New Local Collision

\begin{tabular}{|c|c|c|c|c|}
\hline \multirow{2}{*}{$\begin{array}{l}\text { Chaining } \\
\text { variables }\end{array}$} & \multicolumn{4}{|c|}{ Conditions on bits } \\
\hline & $31-24$ & $23-16$ & $15-8$ & $7-0$ \\
\hline$b_{1}$ & $1 \overline{0}-\cdots \cdots$ & $\overline{\mathrm{a}}-\ldots$ & $-\cdots-\cdots a-$ & $a---\bar{a}-01$ \\
\hline$b_{2}$ & $1 \overline{0}-\overline{0}-\bar{a}-$ & $----0--$ & $---a-1$ & $0-\cdots-\cdots 1$ \\
\hline$b_{3}$ & $1 \overline{1}-\overline{1} \bar{a}-\bar{a} c$ & $\overline{0}-\mathrm{a}$ a $1-\overline{\mathrm{a}} \overline{\mathrm{a}}$ & $\overline{\mathrm{a}} \overline{\mathrm{a}} \overline{\mathrm{a}} \overline{\mathrm{a}} 1 \overline{\mathrm{a}} 0$ & $0--1-10$ \\
\hline$b_{4}$ & $1-\bar{a}-\overline{1} \overline{0} \overline{1}$ & a a $100-\overline{0} 1$ & $\overline{1} \overline{1} \overline{1} \overline{1} 0 \overline{11} \mathrm{a}$ & 1 a a a a - - \\
\hline$b_{5}$ & $\mathrm{a}---\overline{0} \overline{0} \overline{0}$ & $11000 \mathrm{a} 10$ & 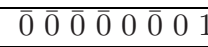 & $111111 \ldots$ \\
\hline$b_{6}$ & $0-\overline{0}-\overline{1}-\overline{1} 1$ & 11111100011 & $\overline{1} \overline{1} \overline{1}-\overline{100}$ & $000000 \mathrm{a} a-$ \\
\hline$b_{7}$ & $0-1--11$ & $00-010--$ & $-\cdots---01$ & $11111111-$ \\
\hline$b_{8}$ & $1-\cdots-a c$ & $00-101--$ & $---0--00$ & $\begin{array}{llllllll}0 & 0 & 0 & 0 & 1 & 0 & 0 & -\end{array}$ \\
\hline$\overline{b_{9}}$ & $0 \mathrm{a} a-\mathrm{a} a 0$ & $01-\cdots$ & $---a--11$ & 111011111 \\
\hline$b_{10}$ & $\begin{array}{llllllllllll}0 & 1 & 1 & - & 1 & 0 & 0 & -\end{array}$ & $11--\bar{a}--$ & $--1-\overline{0} \overline{0}$ & $-\cdots-\cdots-$ \\
\hline$b_{11}$ & $000-110$ & $11-\cdots$ & $---0-1 \overline{1}$ & $-\cdots-\cdots-\cdots$ \\
\hline$b_{12}$ & $\begin{array}{llllllll}0 & 1 & 1 & \text { a } & 0 & 0 & 1\end{array}$ & $-\cdots--1-\cdots$ & $---1-\overline{1} \overline{0}$ & $---\cdots-\cdots$ \\
\hline$b_{13}$ & $--10--0$ & $----1-$ & $---0-\overline{0} \overline{0}$ & $-\cdots-\cdots-\cdots$ \\
\hline$b_{14}$ & $--00--0$ & $----\bar{a}-\cdots$ & $---0-1 \overline{1}$ & $-\cdots-\cdots$ \\
\hline$b_{15}$ & $a-11 b-1$ & $-\cdots-\cdots-b$ & $-\cdots-\cdots-\cdots$ & $-\cdots-\cdots-\cdots$ \\
\hline$b_{16}$ & $1--\mathrm{a} \overline{0}-\cdots$ & $---\bar{c}-\cdots$ & $-\cdots-\cdots$ & $-\cdots-\cdots$ \\
\hline$b_{17}$ & $\mathrm{~b}--0-\cdots$ & $---\bar{a}-\cdots$ & $-\cdots-\cdots-\cdots$ & $-\cdots-\cdots-\cdots$ \\
\hline$b_{18}$ & $\mathrm{~b}--\mathrm{c}-\cdots$ & $-\cdots-\cdots-\cdots$ & $----\cdots$ & $-\cdots-\cdots$ \\
\hline$b_{19}$ & $---\mathrm{a}-\cdots$ & $--\cdots-\cdots$ & $-\cdots-\cdots$ & $-\cdots-\cdots$ \\
\hline$b_{20}$ & $\mathrm{a}-\cdots-\cdots$ & $-\cdots-\cdots$ & - - - - - - & - - - - - - \\
\hline$b_{21}$ & $0-\cdots \cdots$ & $-b-----$ & $-\cdots-\cdots$ & $-\cdots-\cdots-\cdots$ \\
\hline$b_{22}$ & $\mathrm{c}-\cdots-\cdots$ & $-\cdots-\cdots-\cdots$ & $-\cdots-\cdots$ & ------ \\
\hline$b_{23}$ & $\mathrm{a}-\cdots-\cdots$ & $--\cdots-\cdots$ & $-\cdots-\cdots$ & $-----\cdots$ \\
\hline$b_{24}$ & $-\cdots-\cdots-\cdots$ & $-\cdots-\cdots-\cdots$ & $-\cdots-\cdots$ & $---\cdots-\cdots$ \\
\hline$\cdots$ & & & & \\
\hline$b_{33}$ & $0 \ldots \cdots$ & $-----\cdots$ & $-\cdots-\cdots$ & $-\cdots-\cdots-1$ \\
\hline & & & & \\
\hline
\end{tabular}

The notation ' 0 ' stands for the conditions $b_{i, j}=0$, the notation ' 1 ' stands for the conditions $b_{i, j}=1$, the notation 'a' stands for the conditions $b_{i, j}=b_{i-1, j}$, 'b' stands for the condition $b_{i, j} \neq b_{i-1, j}$ and 'c' stands for the condition $b_{i, j}=b_{i-2, j}$. Conditions without upper bar are sufficient conditions for the new differential path. Conditions with upper bar are extra conditions that are introduced in Appendix D.

\section{List of Message Modification Procedures}

Table 9 to Table 20 show the procedure for advanced message modification for each condition. These procedures are executed only if the target condition is not satisfied. Several procedures need to satisfy "extra conditions (EC)" in advance. ECs guarantee that each modification procedure is executed without breaking other sufficient conditions. ECs in the first round are set together with sufficient conditions in the first round by basic message modification. 
We explain how these procedures work, by explaining Table 10 as an example. Before we execute Table [10, we check whether the target sufficient condition " $b_{17,28}=0$ " is satisfied or not. If it's satisfied, do nothing. Otherwise, execute procedures shown in Table 10. In the table, the first and second column show the rounds and steps where we are looking on. The third column shows the procedure to be executed. The last column shows how differences are used in each calculation. In case of Table 10, we first modify $m_{0}$. Then, by considering the expression in step 1 , we modify $b_{1}$. We make sure that a carry doesn't occur in $b_{1}$. Therefore, if $b_{1,28}=0$ we choose "+," otherwise, we choose "-." After that, to make sure that the result of step 2 keeps unchanged, we modify $m_{1}$. In 3 rd step, from the property of $f$, if $b_{2,28}$ is fixed to be 0 , the result of step 3 keeps unchanged. Therefore, we set the extra condition $" b_{2,28}=0 . "$ Step 4 and 5 are the almost same. After step 5 is calculated, all differences in the first round are cancelled. Since $m_{0}$ is modified, step 17 in the second round is also modified. From the expression of $b_{17}, b_{17}$ has difference $2^{28}$, and this difference flips the value of $b_{17,28}$. Therefore, the sufficient condition is satisfied.

In this section, " $b_{i} \leftarrow$ standard computation" means computing $b_{i} \leftarrow\left(a_{i-1}+\right.$ $\left.f\left(b_{i-1}, c_{i-1}, d_{i-1}\right)+m_{k}+t\right) \lll s_{i}$. " $m_{i} \leftarrow$ inverse computation" means computing $m_{i} \leftarrow\left(b_{i} \ggg s_{i}\right)-a_{i-1}-f\left(b_{i-1}, c_{i-1}, d_{i-1}\right)-t$.

Table 9. Modification Procedure for Extra Condition " $b_{17,19}=c_{17,19}$ "

\begin{tabular}{|c|c|c|c|}
\hline Round & Step & Actual procedure & Expression with difference in each step \\
\hline 1R & 16 & $m_{15} \leftarrow m_{15} \pm 2^{29}$ & $b_{16} \leftarrow\left(a_{15}+f\left(b_{15}, c_{15}, d_{15}\right)+m_{15} \pm 2^{29}+t\right) \lll 19$ \\
& & $\begin{array}{c}b_{16} \leftarrow b_{16} \pm 2^{16} \\
\left.\text { (Take sign to avoid } b_{16} \text { carry. }\right)\end{array}$ & \\
\hline $2 \mathrm{R}$ & 17 & $\begin{array}{c}\text { EC: "c } c_{16,16} \neq d_{16,16 "} \text { " } \\
b_{17} \leftarrow \text { standard computation }\end{array}$ & $b_{17} \leftarrow\left(a_{16}+f\left(b_{16}[ \pm 16], c_{16}, d_{16}\right)+m_{0}+t\right) \lll 3$ \\
\hline
\end{tabular}

Table 10. Modification Procedure for " $b_{17,28}=0$ "

\begin{tabular}{|c|c|c|c|}
\hline Round & Step & Actual procedure & Expression with difference in each step \\
\hline \multirow{7}{*}{$1 \mathrm{R}$} & 1 & $m_{0} \leftarrow m_{0} \pm 2^{25}$ & $b_{1} \leftarrow\left(a_{0}+f\left(b_{0}, c_{0}, d_{0}\right)+m_{0} \pm 2^{25}+t\right) \lll 3$ \\
& & $\begin{array}{c}b_{1} \leftarrow b_{1} \pm 2^{28} \\
\left.\text { (Take sign to avoid } b_{1} \text { carry. }\right)\end{array}$ & \\
\cline { 2 - 5 } & 2 & $m_{1} \leftarrow$ inverse computation & $b_{2} \leftarrow\left(a_{1}+f\left(b_{1}[ \pm 28], c_{1}, d_{1}\right)+m_{1}+t\right) \lll 7$ \\
\cline { 2 - 4 } & 3 & EC: " $b_{2,28}=0$ " & $b_{3} \leftarrow\left(a_{2}+f\left(b_{2}, c_{2}[ \pm 28], d_{2}\right)+m_{2}+t\right) \lll 11$ \\
\cline { 2 - 4 } & 4 & EC: "b $b_{3,28}=1$ " & $b_{4} \leftarrow\left(a_{3}+f\left(b_{3}, c_{3}, d_{3}[ \pm 28]\right)+m_{3}+t\right) \lll 19$ \\
\cline { 2 - 5 } & 5 & $m_{4} \leftarrow m_{4} \mp 2^{2 \delta}$ & $b_{5} \leftarrow\left(a_{4} \pm 2^{2 \delta}+f\left(b_{4}, c_{4}, d_{4}\right)+m_{4} \mp 2^{2 \delta}+t\right) \lll 3$ \\
\hline 2R & 17 & $b_{17} \leftarrow$ standard computation & $b_{17} \leftarrow\left(a_{16}+f\left(b_{16}, c_{16}, d_{16}\right)+m_{0} \pm 2^{25}+t\right) \lll 3$ \\
\hline
\end{tabular}


Table 11. Modification Procedure for " $b_{17,31} \neq b_{16,31}$ "

\begin{tabular}{|c|c|c|c|}
\hline Round & Step & Actual procedure & Expression with difference in each step \\
\hline \multirow[t]{5}{*}{$1 \mathrm{R}$} & 1 & $\begin{array}{c}m_{0} \leftarrow m_{0}+2^{27} \\
b_{1} \leftarrow b_{1}+2^{30} \\
\text { EC: }: " b_{1,30}=0 "\end{array}$ & $b_{1} \leftarrow\left(a_{0}+f\left(b_{0}, c_{0}, d_{0}\right)+m_{0}+2^{27}+t\right) \lll 3$ \\
\hline & 2 & $m_{1} \leftarrow$ inverse operation & $b_{2} \leftarrow\left(a_{1}+f\left(b_{1}[+30], c_{1}, d_{1}\right)+m_{1}+t\right) \lll 7$ \\
\hline & 3 & $\mathrm{EC}: " b_{2,30}=0 "$ & $b_{3} \leftarrow\left(a_{2}+f\left(b_{2}, c_{2}[+30], d_{2}\right)+m_{2}+t\right) \lll 11$ \\
\hline & 4 & EC: " $b_{3,30}=1 "$ & $b_{4} \leftarrow\left(a_{3}+f\left(b_{3}, c_{3}, d_{3}[+30]\right)+m_{3}+t\right) \lll 19$ \\
\hline & 5 & $m_{4} \leftarrow m_{4}-2^{30}$ & $b_{5} \leftarrow\left(a_{4}+2^{30}+f\left(b_{4}, c_{4}, d_{4}\right)+m_{4}-2^{30}+t\right) \lll 3$ \\
\hline \multirow[t]{2}{*}{$1-2 \mathrm{R}$} & 16 & $\begin{array}{l}m_{15} \leftarrow m_{15}+2^{8} \\
\text { EC: } " b_{16,27}=0 "\end{array}$ & $b_{16} \leftarrow\left(a_{15}+f\left(b_{15}, c_{15}, d_{15}\right)+m_{15}+2^{8}+t\right) \lll 19$ \\
\hline & 17 & $\begin{array}{c}b_{17} \leftarrow \text { standard operation } \\
\text { EC: } " c_{16,27} \neq d_{16,27} "\end{array}$ & $b_{17} \leftarrow\left(a_{16}+f\left(b_{16}[+27], c_{16}, d_{16}\right)+m_{0}+2^{2 t}+t\right) \lll 3$ \\
\hline
\end{tabular}

Table 12. Modification Procedure for " $b_{18,28}=0 "$

\begin{tabular}{|c|c|c|c|}
\hline Round & Step & Actual procedure & Expression with difference in each step \\
\hline \multirow[t]{5}{*}{$1 \mathrm{R}$} & 2 & $\begin{array}{c}m_{1} \leftarrow m_{1} \pm 2^{16} \\
b_{2} \leftarrow b_{2} \pm 2^{23} \\
\text { (Take sign to avoid } b_{2} \text { carry.) }\end{array}$ & $b_{2} \leftarrow\left(a_{1}+f\left(b_{1}, c_{1}, d_{1}\right)+m_{1} \pm 2^{16}+t\right) \lll 7$ \\
\hline & 3 & EC: " $c_{2,23}=d_{2,23}$ " & $b_{3} \leftarrow\left(a_{2}+f\left(b_{2}[ \pm 23], c_{2}, d_{2}\right)+m_{2}+t\right) \lll 11$ \\
\hline & 4 & EC: $" b_{3,23}=0 "$ & $b_{4} \leftarrow\left(a_{3}+f\left(b_{3}, c_{3}[ \pm 23], d_{3}\right)+m_{3}+t\right) \lll 19$ \\
\hline & 5 & $\begin{array}{l}m_{4} \leftarrow m_{4} \mp 2^{23} \\
\mathrm{EC}: " b_{4,23}=0 "\end{array}$ & $b_{5} \leftarrow\left(a_{4}+f\left(b_{4}, c_{4}, d_{4}[ \pm 23]\right)+m_{4} \mp 2^{23}+t\right) \lll 3$ \\
\hline & 6 & $m_{5} \leftarrow m_{5} \mp 2^{23}$ & $b_{6} \leftarrow\left(a_{5} \pm 2^{23}+f\left(b_{5}, c_{5}, d_{5}\right)+m_{5} \mp 2^{23}+t\right) \lll 7$ \\
\hline $2 \mathrm{R}$ & 17 & $b_{18} \leftarrow$ standard computation & $b_{18} \leftarrow\left(a_{17}+f\left(b_{17}, c_{17}, d_{17}\right)+m_{4} \mp 2^{23}+t\right) \lll 5$ \\
\hline
\end{tabular}

Table 13. Modification Procedure for " $b_{18,31} \neq b_{17,31}$ "

\begin{tabular}{|c|c|c|c|}
\hline Round & Step & Actual procedure & Expression with difference in each step \\
\hline \multirow[t]{5}{*}{$1 \mathrm{R}$} & 5 & $\begin{array}{c}m_{4} \leftarrow m_{4} \pm 2^{26} \\
b_{5} \leftarrow b_{5} \pm 2^{29} \\
\text { (Take sign to avoid } b_{5} \text { carry.) }\end{array}$ & $b_{5} \leftarrow\left(a_{4}+f\left(b_{4}, c_{4}, d_{4}\right)+m_{4} \pm 2^{26}+t\right) \lll 3$ \\
\hline & 6 & EC: $" c_{5,29}=d_{5,29 "}$ & $b_{6} \leftarrow\left(a_{5}+f\left(b_{5}[ \pm 29], c_{5}, d_{5}\right)+m_{5}+t\right) \lll 7$ \\
\hline & 7 & EC: " $b_{6,29}=0 "$ & $b_{7} \leftarrow\left(a_{6}+f\left(b_{6}, c_{6}[ \pm 29], d_{6}\right)+m_{6}+t\right) \lll 11$ \\
\hline & 8 & EC: " $b_{7,29}=1 "$ & $b_{8} \leftarrow\left(a_{7}+f\left(b_{7}, c_{7}, d_{7}[ \pm 29]\right)+m_{7}+t\right) \lll 19$ \\
\hline & 9 & $m_{8} \leftarrow m_{8} \mp 2^{29}$ & $b_{9} \leftarrow\left(a_{8} \pm 2^{29}+\left(f\left(b_{8}, c_{8}, d_{8}\right)+m_{8} \mp 2^{29}+t\right) \lll 3\right.$ \\
\hline $2 \mathrm{R}$ & 17 & $b_{18} \leftarrow$ standard computation & $b_{18} \leftarrow\left(a_{17}+f\left(b_{17}, c_{17}, d_{17}\right)+m_{4} \pm 2^{26}+t\right) \lll 5$ \\
\hline
\end{tabular}

Table 14. Modification Procedure for " $b_{19,28}=b_{18,28}$ "

\begin{tabular}{|c|c|c|c|}
\hline Round & Step & Actual procedure & Expression with difference in each step \\
\hline \multirow{4}{*}{$1-2 \mathrm{R}$} & 15 & $\begin{array}{c}m_{14} \leftarrow m_{14} \pm 2^{8} \\
b_{15} \leftarrow b_{15} \pm 2^{19}\end{array}$ & $b_{15} \leftarrow\left(a_{14}+f\left(b_{14}, c_{14}, d_{14}\right)+m_{14} \pm 2^{8}+t\right) \lll 11$ \\
& & $\begin{array}{c}\left.\text { (Take sign to avoid } b_{15} \text { carry. }\right) \\
\end{array}$ & \\
\cline { 2 - 4 } & 16 & EC: " $c_{15,19}=d_{15,19}$ " & $b_{16} \leftarrow\left(a_{15}+f\left(b_{15}[ \pm 19], c_{15}, d_{15}\right)+m_{15}+t\right) \lll 19$ \\
\cline { 2 - 4 } & 17 & EC: " $b_{16,19}=d_{16,19}$ " & $b_{17} \leftarrow\left(a_{16}+f\left(b_{16}, c_{16}[ \pm 19], d_{16}\right)+m_{0}+t\right) \lll 3$ \\
\cline { 2 - 4 } & 18 & EC: " $b_{17,19}=c_{17,19}$ " & $b_{18} \leftarrow\left(a_{17}+f\left(b_{17}, c_{17}, d_{17}[ \pm 19]\right)+m_{4}+t\right) \lll 5$ \\
\cline { 2 - 5 } & 19 & $b_{19} \leftarrow$ standard computation & $b_{19} \leftarrow\left(a_{18} \pm 2^{19}+f\left(b_{18}, c_{18}, d_{18}\right)+m_{8}+t\right) \lll 9$ \\
\hline
\end{tabular}


Table 15. Modification Procedure for " $b_{20,31}=b_{19,31} "$

\begin{tabular}{|c|c|c|c|}
\hline round & step & Actual procedure & Expression with difference in each step \\
\hline \multirow[t]{5}{*}{$1 \mathrm{R}$} & 11 & $\begin{array}{c}m_{10} \leftarrow m_{10} \pm 2^{7} \\
b_{11} \leftarrow b_{11} \pm 2^{18} \\
\text { take sign to avoid } b_{11} \text { carry }\end{array}$ & $b_{11} \leftarrow\left(a_{10}+f\left(b_{10}, c_{10}, d_{10}\right)+m_{10} \pm 2^{7}+t\right) \lll 11$ \\
\hline & 12 & EC: " $c_{11,18}=d_{11,18}$ " & $b_{12} \leftarrow\left(a_{11}+f\left(b_{11}[ \pm 18], c_{11}, d_{11}\right)+m_{11}+t\right) \lll 19$ \\
\hline & 13 & $m_{12} \leftarrow m_{12} \mp 2^{18}$ & $b_{13} \leftarrow\left(a_{12}+f\left(b_{12}, c_{12}[ \pm 18], d_{12}\right)+m_{12} \mp 2^{18}+t\right) \lll 3$ \\
\hline & 14 & $\begin{array}{l}\text { EC: } " b_{12,18}=1 " \\
\text { EC: } " b_{13,18}=1 "\end{array}$ & $b_{14} \leftarrow\left(a_{13}+f\left(b_{13}, c_{13}, d_{13}[ \pm 18]\right)+m_{13}+t\right) \lll 7$ \\
\hline & 15 & $m_{14} \leftarrow m_{14} \mp 2^{18}$ & $b_{15} \leftarrow\left(a_{14} \pm 2^{18}+f\left(b_{14}, c_{14}, d_{14}\right)+m_{14} \mp 2^{18}+t\right) \lll 11$ \\
\hline $2 \mathrm{R}$ & 20 & $b_{20} \leftarrow$ standard operation & $b_{20} \leftarrow\left(a_{19}+f\left(b_{19}, c_{19}, d_{19}\right)+m_{12} \mp 2^{18}+t\right) \lll 13$ \\
\hline
\end{tabular}

Table 16. Modification Procedure for Extra Condition " $b_{21,22} \neq b_{20,22}$ "

\begin{tabular}{|c|c|c|c|}
\hline Round & Step & Actual procedure & Expression with difference in each step \\
\hline \multirow[t]{5}{*}{$1 \mathrm{R}$} & 12 & $\begin{array}{c}m_{11} \leftarrow m_{11} \pm 2^{22} \\
b_{12} \leftarrow b_{12} \pm 2^{9} \\
\text { EC: " } b_{12,10}=1 ", " b_{12,9}=0 " \\
\left.\text { (if sign is }+, b_{3}[9], \text { else } b_{3}[-10,9] .\right)\end{array}$ & $\begin{aligned} b_{12} & \leftarrow\left(a_{11}+f\left(b_{11}, c_{11}, d_{11}\right)+\right. \\
& \left.+m_{11} \pm 2^{22}+t\right) \lll 19\end{aligned}$ \\
\hline & 13 & $\begin{array}{c}m_{12} \leftarrow m_{12} \mp 2^{9} \\
\text { (Take sign to avoid } b_{20} \text { carry.) } \\
\text { EC: " } c_{12,10}=1 ", " d_{12,10}=0 " \\
\text { " } c_{12,9}=1 ", " d_{12,9}=0 "\end{array}$ & $\begin{array}{c}b_{13} \leftarrow\left(a_{12}+f\left(b_{12}[9] /[-10,9], c_{12}, d_{12}\right)\right. \\
\left.+m_{12} \mp 2^{9}+t\right) \lll 3\end{array}$ \\
\hline & 14 & EC: " $b_{13,10}=0 ", " b_{13,9}=1 "$ & $\begin{array}{c}b_{14} \leftarrow\left(a_{13}+f\left(b_{13}, c_{13}[9] /[-10,9], d_{13}\right)\right. \\
\left.+m_{13}+t\right) \lll 7\end{array}$ \\
\hline & 15 & EC: " $b_{14,10}=1 ", " b_{14,9}=1 "$ & $\begin{array}{c}b_{15} \leftarrow\left(a_{14}+f\left(b_{14}, c_{14}, d_{14}[9] /[-10,9]\right)\right. \\
\left.+m_{14}+t\right) \lll 11\end{array}$ \\
\hline & 16 & $m_{15} \leftarrow m_{15} \mp 2^{9}$ & $\begin{aligned} b_{16} \leftarrow & \left(a_{15} \pm 2^{9}+f\left(b_{15}, c_{54}, d_{15}\right)\right. \\
& \left.+m_{15} \mp 2^{9}+t\right) \lll 19\end{aligned}$ \\
\hline \multirow[t]{2}{*}{$2 \mathrm{R}$} & 20 & $b_{20} \leftarrow$ standard computation & $\begin{aligned} b_{20} & \leftarrow\left(a_{19}+f\left(b_{19}, c_{19}, d_{19}\right)\right. \\
& \left.+m_{12} \mp 2^{9}+t\right) \lll 13\end{aligned}$ \\
\hline & 21 & $\begin{array}{c}b_{21} \leftarrow \text { standard computation } \\
\left(b_{21,22} \text { is never changed by this calculation }\right)\end{array}$ & $\begin{array}{c}b_{21} \leftarrow\left(a_{20}+f\left(b_{20}[\mp 22], c_{20}, d_{20}\right)\right. \\
\left.+m_{1}+t\right) \lll 3\end{array}$ \\
\hline
\end{tabular}

Table 17. Modification Procedure for " $b_{21,31}=0 "$

\begin{tabular}{|c|c|c|c|}
\hline Round & Step & Actual procedure & Expression with difference in each step \\
\hline \multirow[t]{5}{*}{$1 \mathrm{R}$} & 2 & $\begin{array}{c}m_{1} \leftarrow m_{1} \pm 2^{28} \\
b_{2} \leftarrow b_{2} \pm 2^{3} \\
\text { (Take sign to avoid } b_{2} \text { carry.) }\end{array}$ & $b_{2} \leftarrow\left(a_{1}+f\left(b_{1}, c_{1}, d_{1}\right)+m_{1} \pm 2^{28}+t\right) \lll 7$ \\
\hline & 3 & EC: " $c_{2,3}=d_{2,3}$ " & $b_{3} \leftarrow\left(a_{2}+f\left(b_{2}[ \pm 3], c_{2}, d_{2}\right)+m_{2}+t\right) \lll 11$ \\
\hline & 4 & $m_{3} \leftarrow$ inverse computation & $b_{4} \leftarrow\left(a_{3}+f\left(b_{3}, c_{3}[ \pm 3], d_{3}\right)+m_{3}+t\right) \lll 19$ \\
\hline & 5 & $\mathrm{EC}: " b_{4,3}=1$ " & $b_{5} \leftarrow\left(a_{4}+f\left(b_{4}, c_{4}, d_{4}[ \pm 3]\right)+m_{4}+t\right) \lll 3$ \\
\hline & 6 & $m_{5} \leftarrow m_{5} \mp 2^{3}$ & $b_{6} \leftarrow\left(a_{5} \pm 2^{3}+f\left(b_{5}, c_{5}, d_{5}\right)+m_{5} \mp 2^{3}+t\right) \lll 7$ \\
\hline $2 \mathrm{R}$ & 21 & $b_{21} \leftarrow$ standard computation & $b_{21} \leftarrow\left(a_{20}+f\left(b_{20}, c_{20}, d_{20}\right)+m_{1} \pm 2^{28}+t\right) \lll 3$ \\
\hline
\end{tabular}

Table 18. Modification Procedure for " $b_{22,31}=b_{21,31}$ "

\begin{tabular}{|c|c|c|c|}
\hline Round & Step & Actual procedure & Expression with difference in each step \\
\hline \multirow[t]{5}{*}{$1 \mathrm{R}$} & 3 & $\begin{array}{c}m_{2} \leftarrow m_{2} \pm 2^{15} \\
b_{3} \leftarrow b_{3} \pm 2^{26} \\
\text { (Take sign to avoid } b_{3} \text { carry.) }\end{array}$ & $b_{3} \leftarrow\left(a_{2}+f\left(b_{2}, c_{2}, d_{2}\right)+m_{2} \pm 2^{15}+t\right) \lll 11$ \\
\hline & 4 & EC: " $c_{3,26}=d_{3,26}$ " & $b_{4} \leftarrow\left(a_{3}+f\left(b_{3}[ \pm 26], c_{3}, d_{3}\right)+m_{3}+t\right) \lll 19$ \\
\hline & 5 & EC: " $b_{4,26}=0 "$ & $b_{5} \leftarrow\left(a_{4}+f\left(b_{4}, c_{4}[ \pm 26], d_{4}\right)+m_{4}+t\right) \lll 3$ \\
\hline & 6 & $\begin{array}{l}m_{5} \leftarrow m_{5} \mp 22^{20} \\
\text { EC: }: b_{5,26}=0 "\end{array}$ & $b_{6} \leftarrow\left(a_{5}+f\left(b_{5}, c_{5}, d_{5}[ \pm 26]\right)+m_{5}+t\right) \lll 7$ \\
\hline & 7 & $m_{6} \leftarrow m_{6} \mp 2^{26}$ & $b_{7} \leftarrow\left(a_{6} \pm 2^{26}+f\left(b_{6}, c_{6}, d_{6}\right)+m_{6} \mp 2^{26}+t\right) \lll 11$ \\
\hline $2 \mathrm{R}$ & 21 & $b_{22} \leftarrow$ standard computation & $b_{22} \leftarrow\left(a_{21}+f\left(b_{21}, c_{21}, d_{21}\right)+m_{5} \mp 2^{26}+t\right) \lll 5$ \\
\hline
\end{tabular}


Table 19. Modification Procedure for " $b_{23,31}=b_{22,31}$ "

\begin{tabular}{|c|c|c|c|}
\hline Round & Step & Actual procedure & Expression with difference in each step \\
\hline \multirow[t]{5}{*}{$1 \mathrm{R}$} & 4 & $\begin{array}{c}m_{3} \leftarrow m_{3} \pm 2^{30} \\
b_{4} \leftarrow b_{4} \pm 2^{17} \\
\text { (Take sign to avoid } b_{4} \text { carry.) }\end{array}$ & $b_{4} \leftarrow\left(a_{3}+f\left(b_{3}, c_{3}, d_{3}\right)+m_{3} \pm 2^{30}+t\right) \lll 19$ \\
\hline & 5 & EC: $" c_{4,17}=d_{4,17} "$ & $b_{5} \leftarrow\left(a_{4}+f\left(b_{4}[ \pm 17], c_{4}, d_{4}\right)+m_{4}+t\right) \lll 3$ \\
\hline & 6 & $\begin{array}{l}m_{5} \leftarrow m_{5} \mp 22^{17} \\
\text { EC: } " b_{5,17}=1 "\end{array}$ & $b_{6} \leftarrow\left(a_{5}+f\left(b_{5}, c_{5}[ \pm 17], d_{5}\right)+m_{5} \mp 2^{17}+t\right) \lll 7$ \\
\hline & 7 & EC: " $b_{6,17}=1 "$ & $b_{7} \leftarrow\left(a_{6}+f\left(b_{6}, c_{6}, d_{6}[ \pm 17]\right)+m_{6}+t\right) \lll 11$ \\
\hline & 8 & $m_{7} \leftarrow m_{7} \mp 2^{17}$ & $b_{8} \leftarrow\left(a_{7} \pm 2^{17}+f\left(b_{7}, c_{7}, d_{7}\right)+m_{7} \mp 2^{17}+t\right) \lll 19$ \\
\hline \multirow[t]{2}{*}{$2 \mathrm{R}$} & 22 & $\begin{array}{c}b_{22} \leftarrow \text { standard computation } \\
\text { (This breaks SC on } b_{22,31} \\
\text { with probability } 2^{-9} \text {.) }\end{array}$ & $b_{22} \leftarrow\left(a_{21}+f\left(b_{21}, c_{21}, d_{21}\right)+m_{5} \mp 2^{17}+t\right) \lll 5$ \\
\hline & 23 & $\begin{array}{c}b_{23} \leftarrow \text { standard computation } \\
\text { EC: } " c_{22,22} \neq d_{22,22} "\end{array}$ & $b_{23} \leftarrow\left(a_{22}+f\left(b_{22}[\mp 22], c_{22}, d_{22}\right)+m_{9}+t\right) \lll 9$ \\
\hline
\end{tabular}

Table 20. Modification Procedure for Starting Collision Search from Middle of the Second Round

In order to satisfy a condition " $b_{33,31}=0$ " in the third round, we modify the value of chaining variable in step 29 , which is a late step of the second round, This difference will propagate until step 33 , and can flip the value of $b_{33,31}$. In this modification, we need to set several extra conditions. We can set all extra conditions when $i=0,9,11,26,28,29,30,31$. Therefore, we can make $2^{8}$ values for $b_{29}$ that satisfy all sufficient conditions in previous steps. Since, we don't control how the difference is propagated from step 29 to 33, the probability of satisfying " $b_{33,31}=0$ " is not 1 . However, 256 times of trials enable us to quickly find a message that also satisfies " $b_{33,31}=0$."

\begin{tabular}{|c|c|c|c|}
\hline Round & Step & Actual procedure & Expression with difference in each step \\
\hline \multirow[t]{5}{*}{$1 \mathrm{R}$} & 4 & $\begin{array}{c}m_{3} \leftarrow m_{3}-2^{i-3} \\
b_{4} \leftarrow b_{4}-2^{i+16} \\
\text { EC: } " b_{4, i+16}=0 "\end{array}$ & $\begin{array}{c}b_{4} \leftarrow\left(a_{3}+f\left(b_{3}, c_{3}, d_{3}\right)+\right. \\
\left.m_{3}-2^{i-3}+t\right) \lll 19\end{array}$ \\
\hline & 5 & EC: " $c_{4, i+16}=d_{4, i+16 "}$ & $\begin{array}{c}b_{5} \leftarrow\left(a_{4}+f\left(b_{4}[-(i+16)], c_{4}, d_{4}\right)+\right. \\
\left.m_{4}+t\right) \lll 3\end{array}$ \\
\hline & 6 & EC: " $b_{5, i+16}=0 "$ & $\begin{array}{c}b_{6} \leftarrow\left(a_{5}+f\left(b_{5}, c_{5}[-(i+16)], d_{5}\right)+\right. \\
\left.m_{5}+t\right) \lll 7\end{array}$ \\
\hline & 7 & EC: $" b_{6, i+16}=1 "$ & $\begin{array}{c}b_{7} \leftarrow\left(a_{6}+f\left(b_{6}, c_{6}, d_{6}[-(i+16)]\right)+\right. \\
\left.m_{6}+t\right) \lll 11\end{array}$ \\
\hline & 8 & $m_{7} \leftarrow m_{7}+2^{\imath+16}$ & $\begin{aligned} b_{8} \leftarrow\left(a_{7}-2^{i+16}+\left(f\left(b_{7}, c_{7}, d_{7}\right)+\right.\right. \\
\left.m_{7}+2^{i+16}+t\right) \lll 19\end{aligned}$ \\
\hline \multirow[t]{4}{*}{$2 \mathrm{R}$} & 29 & $b_{29} \leftarrow$ standard computation & $\begin{array}{c}b_{29} \leftarrow\left(a_{28}+f\left(b_{28}, c_{28}, d_{28}\right)+\right. \\
\left.m_{3}-2^{i-3}+t\right) \lll 3\end{array}$ \\
\hline & 30 & $b_{30} \leftarrow$ standard computation & \multirow{4}{*}{$\begin{array}{l}\text { Difference propagation is out of control. } \\
\text { Probabilistically flip the value of } b_{33,31} \text {. }\end{array}$} \\
\hline & 31 & $b_{31} \leftarrow$ standard computation & \\
\hline & 32 & $b_{32} \leftarrow$ standard computation & \\
\hline $3 \mathrm{R}$ & 33 & $b_{33} \leftarrow$ standard computation & \\
\hline
\end{tabular}

